\title{
Determination of the NMR Monomer Shift and Dimerization Constant in a Self-associating System by Direct Application of the Least-squares Method
}

\author{
Henry K. S. Tan \\ Department of Applied Chemistry, National Chiao Tung University, Hsinchu, Taiwan, ROC
}

\begin{abstract}
The monomer shift and dimerization constant of a self-associating system have been determined directly by applying the least-squares method to the NMR experimental data. Equations have been obtained in which two analytic methods can be applied. In the first method, a value of the dimerization constant is assumed and by linear regression the error sum of squares (ESS) is calculated. The minimum ESS is located by a five-point searching algorithm. In the second method, non-linear regression analysis of the model equation with the leastsquares method results in a single equation of unknown $K$. A conventional root finding method is then applied from which the values of monomer shift and dimerization constant are determined. The numerical procedures proposed in this paper have been illustrated with an example using previously published experimental NMR data. Results of calculations are discussed.
\end{abstract}

NMR experiments have been widely used in the study of hydrogen bonding, kinetics and equilibrium behaviour of a variety of systems. The determination of the monomer shift and dimerization constant of a self-associating system has been investigated by Purcell et al., ${ }^{1}$ Chen and Shirts ${ }^{2}$ and Chen and Rosenberger. ${ }^{3}$ As pointed out by these authors the determination of the monomer shift by extrapolating experimental data to infinite dilution is not very reliable. Therefore, Purcell et al. applied a direct search procedure, Chen and co-workers proposed both iterative and graphical methods for more accurate determination of the monomer shift and dimerization constant. In this paper we first briefly review the equations and methods used by Chen and co-workers in their studies. ${ }^{2,3}$ We then present alternative methods which are based on the direct application of the least-squares method for the curve fitting of non-linear equations. We also derive the necessary expressions for the direct determination of monomer shift and dimer dissociation constant from the experimental data obtained. An example, using previously published experimental results, is used to illustrate the methods proposed in this paper. In addition, we also include in this work an analytic way of determining monomer shift and dimer constant which is based on Chen and Rosenberger's graphical procedure.

\section{Previous Methods used in the Treatment of Experimental Data}

The observed chemical shift, $\delta$, for a monomer-dimer equilibrium system can be expressed as the weighted average of monomer and dimer shifts ${ }^{2-4}$ with the form of

$$
\delta=\frac{[\mathrm{A}]}{[\mathrm{A}]_{0}} \delta_{\mathrm{m}}+\frac{[\mathrm{A}]_{0}-[\mathrm{A}]}{[\mathrm{A}]_{0}} \delta_{\mathrm{d}}
$$

where $[\mathrm{A}]$ is the equilibrium monomer concentration, $[\mathrm{A}]_{0}$ is the total concentration, while $\delta_{\mathrm{m}}$ and $\delta_{\mathrm{d}}$ are the monomer and dimer shifts, respectively.

The equilibrium constant, $K$, for the self-association of $\mathrm{A}$ to the dimer $\mathrm{A}_{2}$ is defined by

$$
K=\frac{[\mathrm{A}]_{0}-[\mathrm{A}]}{2[\mathrm{~A}]^{2}}
$$

Combining eqn. (1) and (2) and after simplification, the following equation is obtained ${ }^{2,3,5}$

$$
\delta=\delta_{\mathrm{d}}-\left(\frac{\delta_{\mathrm{d}}-\delta_{\mathrm{m}}}{2 K}\right)^{1 / 2}\left(\frac{\delta-\delta_{\mathrm{m}}}{[\mathrm{A}]_{0}}\right)^{1 / 2}
$$

This form of equation has been used by several authors ${ }^{6,7}$ to determine $\delta_{\mathrm{m}}$ and $K$ with the value of $\delta_{\mathrm{m}}$ obtained from extrapolation at dilute concentration. As shown by Chen and Shirts, eqn. (3) can also be transformed to

$$
\begin{aligned}
\delta= & \delta_{\mathrm{m}}+\left(\delta_{\mathrm{d}}-\delta_{\mathrm{m}}\right) \\
& \times\left\{\sqrt{ }\left(1+8 K[\mathrm{~A}]_{0}\right)-1\right\} /\left\{\sqrt{ }\left(1+8 K[\mathrm{~A}]_{0}\right)+1\right\}
\end{aligned}
$$

Note that eqn. (4) is an explicit form for $\delta$ in contrast to the implicit form given in eqn. (3).

The iterative method proposed by Chen and Shirts starts with an assumed value of $\delta_{\mathrm{m}}$ and by applying a quadratic polynomial regression to eqn. (3). The intercept and the limiting slope (corresponding to the first and second coefficient, respectively, in the quadratic polynomial expression) provide an estimate of the value of $K$. This estimate of $K$ is then substituted into eqn. (4) and again a quadratic polynomial regression is carried out for the determination of a more accurate estimate of $\delta_{\mathrm{m}}$. The process continues until the convergence in $\delta_{\mathrm{m}}$ to the desired accuracy is achieved. Although this is a very useful method for determining monomer shift, in view of the inherent iterative calculations required, the use of some other methods may be warranted. Therefore, Chen and Rosenberger proposed a graphical approach for dealing with eqn. (3) or (4). The main argument in this graphical method is that if the correct value of $\delta_{\mathrm{m}}$ is located then linear or quadratic polynomial regression with respect to either eqn. (3) or (4) will lead to an identical quality of curve fitting. In other words, the coefficient of the quadratic term of the polynomial equation approaches zero. Using eqn. (3) and by assuming various values of $\delta_{\mathrm{m}}$, a series of $\delta_{\mathrm{m}} v s . K$ values are generated from both linear and quadratic regressional analysis. Similarly, using eqn. (4) and assumed values of $K$, a series of $K v$ s. $\delta_{\mathrm{m}}$ values can also be obtained with both linear and quadratic regressional analysis. The intersection of $K v s$. $\delta_{\mathrm{m}}$ plots from both linear and quadratic treatment yields the correct values of $\delta_{\mathrm{m}}$ and $K$. This graphical method is very efficient and is an improvement of the iterative scheme used previously by Chen and Shirts. Applying this graphical procedure together with the final numerical refinement can lead 
to a precise determination of values of monomer shift and dimerization constant within the accuracy of the measured spectroscopic data.

\section{Proposed Methods}

To facilitate the derivation of a general expression for directly determining the dimerization equilibrium constant and monomer shift we convert eqn. (3) to the following form

$$
\delta=\delta_{\mathrm{d}}+\left(\delta_{\mathrm{m}}-\delta_{\mathrm{d}}\right)\left\{\sqrt{ }\left(1+8 K[\mathrm{~A}]_{\mathrm{o}}\right)-1\right\} /\left(4 K[\mathrm{~A}]_{0}\right)
$$

Let $a$ denote $\delta_{\mathrm{d}}, b$ denote $\left(\delta_{\mathrm{m}}-\delta_{\mathrm{d}}\right)$ and let the measured $\delta$ be denoted by $Y$, and the concentration $[\mathrm{A}]_{0}$ be denoted by $X$. The error sum of squares from a set values of $\delta_{\mathrm{d}}, \delta_{\mathrm{m}}$ and $K$ is

$$
\mathrm{ESS}=\sum_{i=1}^{N}\left(Y_{i}-\left\{a+b\left[\sqrt{ }\left(1+8 K X_{i}\right)-1\right] /\left(4 K X_{i}\right)\right\}\right)^{2}
$$

where $N$ is the number of data points.

It will be assumed that the three parameters of $\delta_{\mathrm{d}}, \delta_{\mathrm{m}}$ and $K$ are to be determined from eqn. (6) when the ESS is a minimum. By partial differentiation of eqn. (6) with respect to $a, b$ and $K$, and equating the derivatives to zero, we obtain

$$
\begin{aligned}
a & =\frac{\sum Y_{i} \sum\left(G_{i}\right)^{2}-\sum G_{i} \sum G_{i} Y_{i}}{N \sum\left(G_{i}\right)^{2}-\sum G_{i} \sum G_{i}} \\
b & =\frac{N \sum G_{i} Y_{i}-\sum G_{i} \sum Y_{i}}{N \sum\left(G_{i}\right)^{2}-\sum G_{i} \sum G_{i}}
\end{aligned}
$$

where

$$
G_{i}=\left[\sqrt{ }\left(1+8 K X_{i}\right)-1\right] /\left(4 K X_{i}\right)
$$

The value of $K$ corresponding to the minimum ESS can then be determined from the following equation

$$
\begin{aligned}
f(K)= & a \sum\left[\frac{1}{\sqrt{ }\left(1+8 K X_{i}\right)}-G_{i}\right] \\
& +b \sum\left[\frac{1}{\sqrt{ }\left(1+8 K X_{i}\right)}-G_{i}\right] \\
& -\sum \frac{Y_{i}}{\sqrt{ }\left(1+8 K X_{i}\right)}+\sum G_{i} Y_{i}=0
\end{aligned}
$$

Note that in eqn. (10), $a, b$ and $G$ are all functions of $K$ and are defined in eqn. (7)-(9), respectively.

Two methods can be used for determining the values of $K$, $a$ and $b$ which give the minimum ESS. The first method is by relying on eqn. (6)-(9). The second method corresponds to the determination of $K$ which satisfies eqn. (10). In the first method, a value of $K$ is assumed, $G_{i}$ is calculated from eqn. (9), and $a$ and $b$ are then calculated from eqn. (7) and (8). Upon substituting the calculated values of $a, b$ and the assumed value of $K$ into eqn. (6), the corresponding ESS is obtained. The process can then be repeated with other assumed values of $K$. The minimum value of the ESS is located by successive iteration. One systematic way of locating this minimum value of the ESS is by the following procedures. If the minimum ESS is known to lie between $K_{1}$ and $K_{5}$, then if $K_{3}$ denotes the midpoint between $K_{1}$ and $K_{5}$ the calculated values of ESS are such that for $K_{1}<K_{3}<K_{5}$, $\operatorname{ESS}(1)>\operatorname{ESS}(3)<\operatorname{ESS}(5)$.

Corresponding values of the ESS at $K_{2}$ and $K_{4}$ are then calculated, where $K_{2}$ and $K_{4}$ are the midpoints between $K_{1}$ and $K_{3}$ and between $K_{3}$ and $K_{5}$, respectively. There are three possible outcomes after the calculation of $\operatorname{ESS}(2)$ and
$\operatorname{ESS}(4): \quad$ (i) $\operatorname{ESS}(2)>\operatorname{ESS}(3)<\operatorname{ESS}(4), \quad$ (ii) $\operatorname{ESS}(1)>$ $\operatorname{ESS}(2)<\operatorname{ESS}(3)$, (iii) $\operatorname{ESS}(3)>\operatorname{ESS}(4)<\operatorname{ESS}(5)$. For case (i), the old $\operatorname{ESS}(2)$ and $\operatorname{ESS}(4)$ become the new $\operatorname{ESS}(1)$ and $\operatorname{ESS}(5)$ in the next stage of iterative calculation. For cases (ii) and (iii), the old $\operatorname{ESS}(1), \operatorname{ESS}(2), \operatorname{ESS}(3)$, or $\operatorname{ESS}(3), \operatorname{ESS}(4), \operatorname{ESS}(5)$ becomes the new $\operatorname{ESS}(1), \operatorname{ESS}(3), \operatorname{ESS}(5)$ in the next iteration. In this manner the interval between $K_{1}$ and $K_{5}$ is reduced by half in each cycle of repetitive calculation.

The second method is in essence the determination of the real and physical meaningful value of a root that satisfies a non-linear algebraic equation. In this case, Newton-Raphson, secant, bisection or some other conventional numerical methods for root finding can be applied. The secant method is preferred if the derivative expression is too complicated to evaluate with the Newton-Raphson method.

In this paper, our primary aim is to suggest alternative methods to the previous ones used by Chen and Shirt ${ }^{2}$ and Chen and Rosenberger. ${ }^{3}$ However, we must point out that the graphical method proposed by Chen and Rosenberger is a very ingenious way of effectively determining $K$ and $\delta_{\mathrm{m}}$ without resorting to a complete regressional analysis which leads to the derivation of eqn. (10). Utilizing Chen and Rosenberger's idea we formulate an analytic expression which can aid us in the determination of $\delta_{\mathrm{m}}$ and $K$. In doing so, plots of $K v s$. $\delta_{\mathrm{m}}$ from both linear and quadratic regression can be omitted. The analytic expression is obtained based on the fact that when the correct $K$ or $\delta_{m}$ is located, the quadratic term coefficient for the quadratic polynomial equation should approach zero. This criterion differs from that of Chen and Rosenberger, since in their method the quadratic term does not have to approach zero as long as the value of $\delta_{\mathrm{m}}$ or $K$ from both linear and quadratic regression converges.

Under the criterion that the quadratic term coefficient should equal zero when the correct $\delta_{\mathrm{m}}$ or $K$ is located, we have, after solving for the quadratic term coefficient, $C$, from quadratic polynomial regression by the method of determinants,

$$
\begin{aligned}
C= & f\left(Z_{i}\right)=\left[\sum Z_{i} \sum Z_{i}^{3}-\sum\left(Z_{i}\right)^{2}\right] \sum Y_{i} \\
& +\left[\sum Z_{i} \sum Z_{i}^{2}-N \sum Z_{i}^{3}\right] \sum Z_{i} Y_{i} \\
& +\left[N \sum Z_{i}^{2}-\left(\sum Z_{i}\right)^{2}\right] \sum Y_{i} Z_{i}^{2}=0
\end{aligned}
$$

where

$$
Z_{i}=f\left(\delta_{\mathrm{m}}\right)=\left(\frac{Y_{i}-\delta_{\mathrm{m}}}{X_{i}}\right)^{1 / 2}
$$

if eqn. (3) is used,

$$
Z_{i}=f(K)=\frac{\sqrt{ }\left(1+8 K X_{i}\right)-1}{\sqrt{ }\left(1+8 K X_{i}\right)+1}
$$

if eqn. (4) is used. The validity of eqn. (11) is, of course, subject to the condition that

$$
\begin{aligned}
N \sum Z_{i}^{2} \sum Z_{i}^{4}+2 \sum & Z_{i} \sum Z_{i}^{2} \sum Z_{i}^{3} \\
& \neq\left(\sum Z_{i}^{2}\right)^{3}+N\left(\sum Z_{i}^{3}\right)^{2}+\sum Z_{i}^{4}\left(\sum Z_{i}\right)^{2}
\end{aligned}
$$

As in the case of eqn. (10), any standard numerical methods for root finding can be applied in eqn. (11) to solve for $K$ or $\delta_{\mathrm{m}}$. Again the secant method which avoids the need for obtaining the derivative expression is more convenient to apply than if the Newton-Raphson method is used.

Note that in eqn. (10), the root, $K$, obtained corresponds to the minimum ESS attainable. On the other hand, eqn. (11) indicates that both linear and quadratic polynomial regres- 
sion lead to the convergence of a set of values of $K, \delta_{\mathrm{m}}$ and $\delta_{\mathrm{d}}$. It should also be pointed out that if the implicit form of eqn. (3) is used, the values of $K, \delta_{\mathrm{m}}$ and $\delta_{\mathrm{d}}$ and the corresponding ESS will be different from those determined from using the explicit form of eqn. (4).

As pointed out by Chen and Shirts, ${ }^{2}$ not all the selfassociating systems are so simple that their equilibrium relationship can be easily depicted by eqn. (2). They have made references to previous studies ${ }^{8,9}$ and believe that for fairly dilute solutions and for systems such as hindered phenols and lactams, the monomer-dimer equilibrium is dominant because of geometric preferences. Thus for these systems, the methods proposed in this paper may be useful. In carrying out the numerical treatment of the experimental data as outlined in this work, we must make the prerequisite assumptions that the value of $K$ is unique and that the criteria of $\delta_{\mathrm{d}}>\delta>\delta_{\mathrm{m}}$ or $\delta_{\mathrm{m}}>\delta>\delta_{\mathrm{d}}$ must be met.

\section{Results and Discussion}

To illustrate the use of the proposed method we employ the experimental NMR data for solutions of $\delta$-valerolactam in $\mathrm{CDCl}_{3}$ as reported by Purcell et al. ${ }^{1}$ These spectral data were obtained with a Varian DA-60 IL spectrometer and with the use of tetramethylsilane as internal reference. Table 1 gives the original data in which the solute concentration is expressed in terms of mole fraction. Following the work of Chen and Shirts, ${ }^{2}$ we have also converted the concentration to $\mathrm{mol} \mathrm{kg} \mathrm{kg}^{-1}$ and the resulting conversions are also given in Table 1 . To carry out linear regression analysis, the value of $K$ is assumed so that $a$ and $b$ can be calculated from eqn. (7) and (8). From the calculated values of $a$ and $b$ the ESS corresponding to the assumed value of $K$ is then determined from eqn. (6). With a series of assumed values of $K$, a plot of ESS $v s$. $K$ can be obtained and is shown in Fig. 1. As can be seen from Fig. 1, the presence of a minimum ESS is evident although the exact location cannot be easily determined by visual inspection. The ESS as shown in Fig. 1 is fairly constant between $K$ values of 5.2 and $5.6 \mathrm{~kg} \mathrm{~mol}^{-1}$. By applying partial regression analysis to eqn. (3), that is assuming a value of $\delta_{m}$ and carrying out the linear regression calculation, plots of ESS $v$ s. $\delta_{\mathrm{m}}$ and ESS vs. $\delta_{\mathrm{d}}$ were also obtained as shown in Fig. 2 and 3. Comparing Fig. 2 and 3, it can be seen that for the range of ESS between 220 and $250 \mathrm{~Hz},{ }^{2}$ the dimer shift

Table 1 Experimental $\mathrm{NMR}$ data of valerolactam in $\mathrm{CDCl}_{3}$, at $306 \mathrm{~K}^{a}$

\begin{tabular}{ccc}
\hline mole fraction & $\begin{array}{c}\text { concentration } \\
/ \mathrm{mol} \mathrm{kg}^{-1}\end{array}$ & $\delta / \mathrm{Hz}^{\mathbf{b}}$ \\
\hline 0.010 & 0.0839 & 382.0 \\
0.020 & 0.1695 & 408.8 \\
0.030 & 0.2569 & 420.7 \\
0.041 & 0.3551 & 426.6 \\
0.052 & 0.4556 & 442.7 \\
0.061 & 0.5396 & 451.0 \\
0.073 & 0.6541 & 444.8 \\
0.083 & 0.7519 & 453.3 \\
0.091 & 0.8316 & 459.5 \\
0.097 & 0.8923 & 458.4 \\
0.150 & 1.4659 & 469.6 \\
0.203 & 2.1157 & 476.9 \\
0.255 & 2.8432 & 484.6 \\
0.300 & 3.5600 & 484.7 \\
0.359 & 4.6522 & 488.3 \\
0.408 & 5.7248 & 491.7 \\
0.449 & 6.7689 & 491.9 \\
0.490 & 7.9809 & 494.3 \\
\hline
\end{tabular}

${ }^{a}$ From ref. $1{ }^{b}$ From TMS.

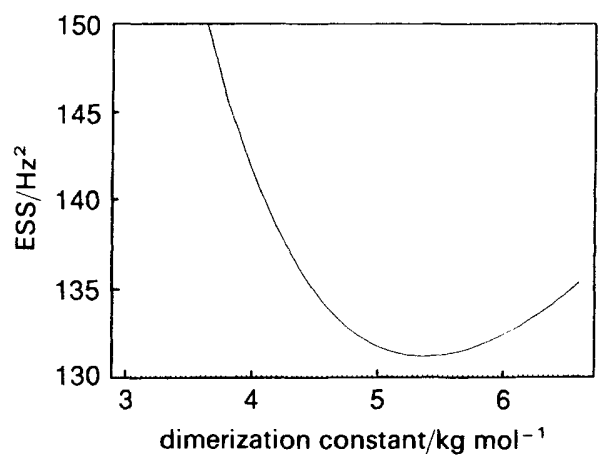

Fig. 1 ESS vs. dimerization constant

does not vary as much as the monomer shift. This is in agreement with Purcell et al.'s finding, which used the direct search procedure, ${ }^{10}$ that the calculated dimer shift is nearly independent of the preset monomer shift. We should also point out that the results of Fig. 1 and 2 do not give the same value for the minimum ESS. This is to be expected since two different forms of the equations, one explicit the other implicit, are used. The explicit type of equation is to be preferred if transformation to a linear form can be accomplished. Graphical output, as shown in Fig. 1, indicates the region in which the minimum ESS and the corresponding $K$ are situated. The exact location, however, must be determined by analytical means. Applying eqn. (6)-(8) and employing the five-point searching algorithm outlined in the previous section, the value of $K$ corresponding to the minimum ESS is calculated. Table 2 gives the iterative calculated results starting with three different initial $K$ ranges. The first is for $K$ between 3 and 7 , the second for $K$ between 1 and 9 and the third for $K$ between 2 and 6 . In the same table the corresponding values of $\delta_{\mathrm{m}}$ and $\delta_{\mathrm{d}}$ at $K_{3}$ for each iterative calculation are also

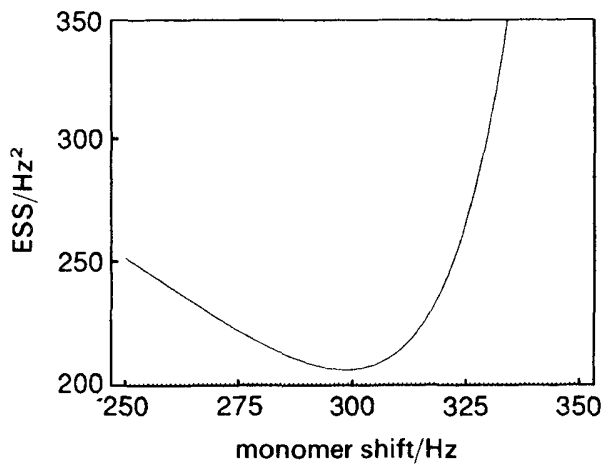

Fig. 2 ESS vs. monomer shift

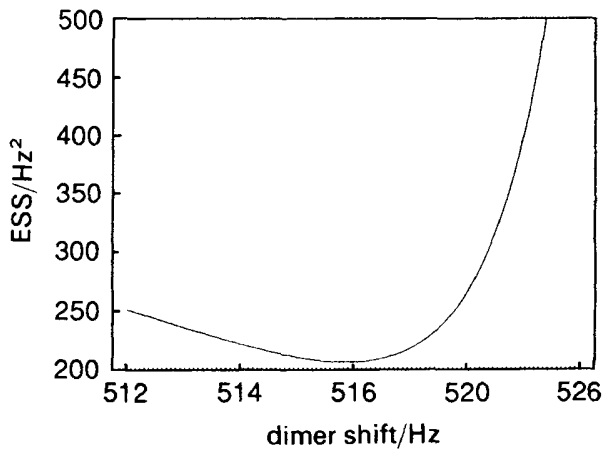

Fig. 3 ESS vs. dimer shift 
Table 2 Calculated results using a five-point searching algorithm for locating the minimum ESS

\begin{tabular}{|c|c|c|c|c|c|c|c|c|}
\hline $\begin{array}{c}\text { iteration } \\
\text { no. }\end{array}$ & $K_{1} / \mathrm{kg} \mathrm{mol}^{-1}$ & $\operatorname{ESS}(1) / \mathbf{H z}^{2}$ & $K_{3} / \mathrm{kg} \mathrm{mol}^{-1}$ & $\mathrm{ESS}(3) / \mathrm{Hz}^{2}$ & $K_{5} / \mathrm{kg} \mathrm{mol}^{-1}$ & $\operatorname{ESS}(5) / \mathrm{Hz}^{2}$ & $\delta_{\mathrm{m} 3} / \mathbf{H z}$ & $\delta_{\mathrm{d} 3} / \mathrm{Hz}$ \\
\hline 1 & 3.000 & 176.485 & 5.000 & 131.718 & 7.000 & 137.828 & 308.99 & 517.31 \\
\hline 2 & 4.000 & 141.557 & 5.000 & 131.718 & 6.000 & 132.398 & 308.99 & 517.31 \\
\hline 3 & 5.000 & 131.718 & 5.500 & 131.214 & 6.000 & 132.398 & 303.03 & 516.61 \\
\hline 4 & 5.000 & 131.718 & 5.250 & 131.212 & 5.500 & 131.214 & 305.98 & 516.94 \\
\hline 5 & 5.250 & 131.212 & 5.375 & 131.155 & 5.500 & 131.214 & 304.50 & 516.77 \\
\hline 6 & 5.313 & 131.168 & 5.375 & 131.155 & 5.438 & 131.171 & 304.50 & 516.77 \\
\hline 7 & 5.344 & 131.158 & 5.375 & 131.155 & 5.406 & 131.160 & 304.50 & 516.77 \\
\hline 8 & 5.359 & 131.156 & 5.375 & 131.155 & 5.391 & 131.157 & 304.50 & 516.77 \\
\hline 1 & 1.000 & 661.437 & 5.000 & 131.718 & 9.000 & 153.942 & 308.99 & 517.31 \\
\hline 2 & 3.000 & 176.485 & 5.000 & 131.718 & 7.000 & 137.828 & 308.99 & 517.31 \\
\hline 3 & 4.000 & 141.557 & 5.000 & 131.718 & 6.000 & 132.398 & 308.99 & 517.31 \\
\hline 4 & 5.000 & 131.718 & 5.500 & 131.214 & 6.000 & 132.398 & 303.03 & 516.61 \\
\hline 5 & 5.000 & 131.718 & 5.250 & 131.212 & 5.500 & 131.214 & 305.98 & 516.94 \\
\hline 6 & 5.250 & 131.212 & 5.375 & 131.155 & 5.500 & 131.214 & 304.50 & 516.77 \\
\hline 7 & 5.313 & 131.168 & 5.375 & 131.155 & 5.438 & 131.171 & 304.50 & 516.77 \\
\hline 1 & 2.000 & 281.500 & 4.000 & 141.557 & 6.000 & 132.398 & 321.92 & 519.08 \\
\hline 2 & 4.000 & 141.557 & 5.000 & 131.718 & 6.000 & 132.398 & 308.99 & 517.31 \\
\hline 3 & 5.000 & 131.718 & 5.500 & 131.214 & 6.000 & 132.398 & 303.03 & 516.61 \\
\hline 4 & 5.000 & 131.718 & 5.250 & 131.212 & 5.500 & 131.214 & 305.98 & 516.94 \\
\hline 5 & 5.250 & 131.212 & 5.375 & 131.155 & 5.500 & 131.214 & 304.50 & 516.77 \\
\hline 6 & 5.313 & 131.168 & 5.375 & 131.155 & 5.438 & 131.171 & 304.50 & 516.77 \\
\hline
\end{tabular}

listed. Note that in these three sample calculations with different initial estimates of $K$ values, convergence to minimum ESS is achieved within six to eight iterations.

In the above procedure, the determination of $K$ is by using partial regression expressions. A complete regression analysis leads to eqn. (10) which can be used for the direct determination of $K$. To find the root of the non-linear equation $f(K)=0$, the secant method is used. Although the NewtonRaphson method can also be applied, evaluation of the derivative expression for $f(K)$ can be complicated and tedious. By the use of the secant method the following expression is employed in the successive iteration

$$
K_{3}=K_{2}-\frac{\left(K_{2}-K_{1}\right) f\left(K_{2}\right)}{f\left(K_{2}\right)-f\left(K_{1}\right)}
$$

where $K_{1}$ and $K_{2}$ are the two initial guesses of $K$ to start the root-finding calculation. After each calculation, the value of $K_{3}$ determined becomes the new $K_{2}$ and the old $K_{2}$ is the new $K_{1}$ in the next iterative calculation. The process is repeated until $K$ converges to the desired accuracy. Table 3 gives the calculated results with three different initial sets of $K_{1}$ and $K_{2}$ values. In all three cases, $K$ is converged to three significant figures within five to seven iterations.

With either five-point minimum ESS searching or direct application of eqn. (10), the value of $K$ is found to be $5.37 \mathrm{~kg}$ $\mathrm{mol}^{-1}$. The corresponding $\delta_{\mathrm{m}}$ and $\delta_{\mathrm{d}}$ are calculated to be

Table 3 Convergence of the root of $f(K)$ using the secant method

\begin{tabular}{cccrcc}
\hline \multicolumn{7}{c}{ initial values of $K_{1}$ and $K_{2}$} \\
\hline$K_{1}$ & $K_{2}$ & $K_{1}$ & \multicolumn{1}{c}{$K_{2}$} & $K_{1}$ & $K_{2}$ \\
3.00 & 4.00 & 7.00 & \multicolumn{1}{c}{8.00} & 1.00 & 2.00 \\
\hline$K$ & $f(K)$ & $K$ & \multicolumn{1}{c}{$f(K)$} & $K$ & $f(K)$ \\
\hline 4.68414 & 0.077193 & 3.92623 & 0.20432 & 2.75318 & 0.56828 \\
5.15510 & 0.021375 & 6.35708 & -0.07247 & 3.67854 & 0.25986 \\
5.33544 & 0.003424 & 5.72065 & -0.02979 & 4.45818 & 0.10953 \\
5.36984 & 0.000186 & 5.27647 & 0.00911 & 5.02612 & 0.03529 \\
5.37182 & 0.000001 & 5.38050 & -0.00081 & 5.29617 & 0.00719 \\
5.37184 & 0.000000 & 5.37205 & -0.00002 & 5.36527 & 0.00061 \\
5.37184 & 0.000000 & 5.37183 & 0.00000 & 5.37171 & 0.00001 \\
& & & & 5.37184 & 0.00000 \\
\hline
\end{tabular}

304.50 and $516.77 \mathrm{~Hz}$, respectively. The minimum ESS is determined to be $131.16 \mathrm{~Hz}^{2}$

By using eqn. (3) with various assumed values of $\delta_{m}$ and applying quadratic polynomial regression, a series of values for the quadratic term coefficient, $C, v s . \delta_{\mathrm{m}}$ is obtained and these are plotted in Fig. 4. Similarly, using eqn. (4) with various assumed values of $K$ and after applying the quadratic polynomial regression, a plot of quadratic term coefficient $v s$. $K$ can be obtained, see Fig. 5. Analytically, the value of either $K$ or $\delta_{\mathrm{m}}$ can be obtained by solving eqn. (11) with a conven-

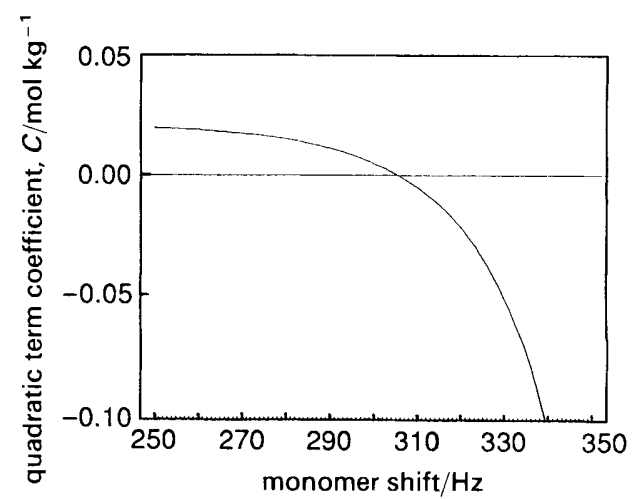

Fig. 4 Quadratic term coefficient vs. monomer shift using eqn. (3) and (11)

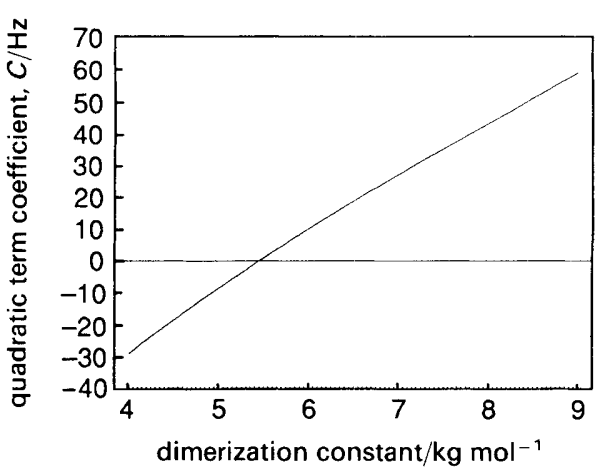

Fig. 5 Quadratic term coefficient vs. dimerization constant using eqn. (4) and (11) 
tional root-finding method. For this example, the calculated $K$ value is $5.44 \mathrm{~kg} \mathrm{~mol}^{-1}$ when the regression is applied to eqn. (4), and $\delta_{\mathrm{m}}$ is calculated to be $305.32 \mathrm{~Hz}$ when regression is applied to eqn. (3).

\section{Conclusions}

In this study we have examined the previous methods used in the determination of monomer shift and dimerization constant for self-associating systems. We have derived a general expression from which the values of $K, \delta_{\mathrm{m}}$ and $\delta_{\mathrm{d}}$ corresponding to the minimum ESS can be determined. By the direct application of a least-squares method, the determination of $K$ reduces to the problem of root finding for a non-linear algebraic equation. In addition, we have introduced in this paper an analytic method which modifies a previous graphical procedure for the determination of monomer shift, $\delta_{\mathrm{m}}$. In contrast to the previous iterative method of Chen and Shirts ${ }^{2}$ and the graphical method employed by Chen and Rosenberger, ${ }^{3}$ we have applied direct minimization of the ESS in the determination of the dimerization constant and chemical shifts for both the monomer and the dimer. We feel that the direct minimization of ESS is a better and more reasonable approach to data treatment. We have proposed an analytical method which is an efficient way of replacing the graphical procedure. Note that the numerical value read from graphs cannot be very accurate and numerical interpolation by analytic means is usually warranted. Therefore, analytical determination is often preferable to graphical means. We must emphasize that the methods proposed in this work may not be applicable for all self-associating systems. It is suggested that more experimental data should be used to examine the utility of the methods and the validity of the model equations used in the data treatment.

The author is indebted to Dr. L. G. Walker of Ryerson Polytechnic University, Toronto, Canada, for his help and assistance in the preparation of this article.

\section{References}

1 J. M. Purcell, H. Susi and J. R. Cavanaugh, Can. J. Chem., 1969, 47, 3655 .

2 J-S. Chen and R. B. Shirts, J. Phys. Chem., 1985, 89, 1643.

3 J-S. Chen and F. Rosenberger, Tetrahedron Lett., 1990, 31, 1975.

4 H. S. Gutowsky and A. Saika, J. Chem. Phys., 1953, 21, 1688.

5 J-L. Dimicoli and C. Helene, J. Am. Chem. Soc., 1973, 95, 1036.

6 J. R. Barrio, F-T. Liu, G. E. Keyser, P. Van Der Lijn and N. J. Leonard, J. Am. Chem. Soc., 1979, 101, 1564.

7 M. Borzo, C. Detellier, P. Laszlo and A. Paris, J. Am. Chem. Soc., 1980, 102, 1124.

8 J. A. Walmseley, J. Phys. Chem., 1978, 82, 2031.

9 B. G. Somers and H. S. Gutowsky, J. Am. Chem. Soc., 1963, 85, 3065.

10 R. Hookes and J. A. Jeeves, J. Assoc. Comput. Mach., 1961, 8, 212.

Paper 4/02996H; Received 19th May, 1994 